\title{
Generalization of Uniqueness Theorems for Entire and Meromorphic Functions
}

\author{
Harina P. Waghamore ${ }^{1}$, N. Shilpa ${ }^{2}$ \\ ${ }^{1}$ Department of Mathematics, Central College Campus, Bangalore University, Bangalore, India \\ ${ }^{2}$ P. G. Department of Mathematics, M. E. S. College, Bangalore, India \\ Email: pree.tam@rediffmail.com, shilpajaikumar@gmail.com
}

Received 3 February 2014; revised 3 March 2014; accepted 10 March 2014

Copyright @ 2014 by authors and Scientific Research Publishing Inc.

This work is licensed under the Creative Commons Attribution International License (CC BY).

http://creativecommons.org/licenses/by/4.0/

c) (i) Open Access

\section{Abstract}

In this paper, we deal with the uniqueness problems on entire and meromorphic functions concerning differential polynomials that share fixed-points. Moreover, we generalise and improve some results of Weichuan Lin, Hongxun Yi, Meng Chao, C. Y. Fang, M. L. Fang and Junfeng xu.

\section{Keywords}

\section{Nevanlinna Theory, Uniqueness, Entire Functions, Meromorphic Functions, Differential} Polynomials, Fixed Points

\section{Introduction}

In this paper, the term "meromorphic" will always mean meromorphic in the complex plane $C$. Let $a$ be a complex number and $\alpha(z)$ be a meromorphic function such that $T(r, \alpha)=o\{T(r, f)\}$. We say $f$ and $g$ share the value $a \mathrm{CM}$, if $f-a$ and $g-a$ assume the same zeros with the same multiplicities; if $f(z)-\alpha(z)$ and $g(z)-\alpha(z)$ assume the same zeros with the same multiplicities, then we say $f(z)$ and $g(z)$ share $\alpha(z)$ $\mathrm{CM}$, especially we say that $f(z)$ and $g(z)$ have the same fixed-points when $\alpha(z)=z$. It is assumed that the reader is familiar with the notations of the Nevanlinna theory that can be found, for instance, in [1]. We denote by $S(r, f)$ any function satisfying

$$
S(r, f)=o\{T(r, f)\}
$$

as $r \rightarrow \infty$, possibly outside of finite measure.

Set

$$
N_{k}\left(r, \frac{1}{f-a}\right)=\bar{N}\left(r, \frac{1}{f-a}\right)+\bar{N}_{(2}\left(r, \frac{1}{f-a}\right)+\cdots+\bar{N}_{(k}\left(r, \frac{1}{f-a}\right) .
$$


It is well known that if $f$ and $g$ share four distinct values CM, then $f$ is a fractional transformation of $g$. In 1997, corresponding to one famous question of Hayman, C. C. Yang and X. H. Hua showed the similar conclusions hold for certain types of differential polynomials when they share only one value. They proved the following result.

Theorem A ([2]). Let $f$ and $g$ be two non-constant meromorphic functions, $n \geq 11$ be an integer and $a \in C-\{0\}$. If $f^{n} f^{\prime}$ and $g^{n} g^{\prime}$ share the value a $C M$, then either $f=d g$ for some $(n+1)^{\text {th }}$ root of unity $d$ or $g(z)=c_{1} \mathrm{e}^{c z}$ and $f(z)=c_{2} \mathrm{e}^{-c z}$, where $c, c_{1}$ and $c_{2}$ are constants and satisfy $\left(c_{1} c_{2}\right)^{n+1} c^{2}=-a^{2}$.

In 2001, M. L. Fang and W. Hong obtained the following result.

Theorem B ([3]). Let $f$ and $g$ be two transcendental entire functions, $n \geq 11$ an integer. If $f^{n}(f-1) f^{\prime}$ and $g^{n}(g-1) g^{\prime}$ share the value $1 C M$, then $f \equiv g$.

Recently, W. C. Lin and H. X. Yi extended the above theorem with respect to fixed point. They proved the following results.

Theorem C ([4]). Let $f$ and $g$ be two transcendental meromorphic functions, $n \geq 12$ an integer. If $f^{n}(f-1) f^{\prime}$ and $g^{n}(g-1) g^{\prime}$ share $z C M$, then either $f(z) \equiv g(z)$ or

$$
f=\frac{(n+2) h\left(1-h^{n+1}\right)}{(n+1)\left(1-h^{n+2}\right)}, \quad g=\frac{(n+2)\left(1-h^{n+1}\right)}{(n+1)\left(1-h^{n+2}\right)}
$$

where $h$ is a nonconstant meromorphic function.

Theorem D ([4]). Let $f$ and $g$ be two transcendental meromorphic functions, $n \geq 13$ an integer. If $f^{n}(f-1)^{2} f^{\prime}$ and $g^{n}(g-1)^{2} g^{\prime}$ share z CM, then $f \equiv g$.

We generalise the above results and prove the following Theorem.

Theorem 1.1 Let $f$ and $g$ be two transcendental meromorphic functions, $n \geq m+11$ an integer. If $f^{n}(f-1)^{m} f^{\prime}$ and $g^{n}(g-1)^{m} g^{\prime}$ share $z \quad C M$ then $f \equiv g$.

For $m=1$, we get Theorem C.

For $m=2$, we get Theorem D.

One may ask the following question, can the nature of the fixed point $z$ be relaxed to $I M$ in the above theorems?

In 2008, Meng Chao answered to the above question and proved the following theorems.

Theorem $\mathbf{E}$ ([5]). Let $f$ and $g$ be two transcendental meromorphic functions, $n \geq 27$ an integer. If $f^{n}(f-1) f^{\prime}$ and $g^{n}(g-1) g^{\prime}$ share $z I M$, then either $f(z) \equiv g(z)$ or

$$
f=\frac{(n+2) h\left(1-h^{n+1}\right)}{(n+1)\left(1-h^{n+2}\right)}, \quad g=\frac{(n+2)\left(1-h^{n+1}\right)}{(n+1)\left(1-h^{n+2}\right)}
$$

where $h$ is a nonconstant meromorphic function.

Theorem $\mathbf{F}([5])$. Let $f$ and $g$ be two transcendental meromorphic functions, $n \geq 28$ an integer. If $f^{n}(f-1)^{2} f^{\prime}$ and $g^{n}(g-1)^{2} g^{\prime}$ share $z$ IM, then $f \equiv g$.

We generalise the above results and prove the following Theorem.

Theorem 1.2 Let $f$ and $g$ be two transcendental meromorphic functions, $n \geq m+26$ an integer. If $f^{n}(f-1)^{m} f^{\prime}$ and $g^{n}(g-1)^{m} g^{\prime}$ share $z$ IM then $f \equiv g$.

For $m=1$, we get $n \geq 24$ which improves Theorem $E$.

For $m=2$, we get $n \geq 28$, we get Theorem F.

In 2002, Fang and Fang [6] proved that there exists a differential polynomial $d$ such that for any pair of nonconstant entire functions $f$ and $g$ we can get $f \equiv g$, if $d(f)$ and $d(g)$ share one value $C M$.

Theorem G ([6]). Let $f$ and $g$ be two nonconstant entire functions, $n \geq 8$ be a positive integer. If $f^{n}(f-1) f^{\prime}$ and $g^{n}(g-1) g^{\prime}$ share $1 C M$, then $f \equiv g$.

In 2004, Lin-Yi [7] and Qiu-Fang [8] proved that Theorem G remains valid for $n \geq 7$.

Theorem $\mathbf{H}$ ([7] [8]). Let $f$ and $g$ be two nonconstant entire functions, $n \geq 7$ be a positive integer. If $f^{n}(f-1) f^{\prime}$ and $g^{n}(g-1) g^{\prime}$ share $1 C M$, then $f \equiv g$.

We generalise the above results and prove the following theorem.

Theorem 1.3 Let $f$ and $g$ be two transcendental entire functions, $n \geq m+6$ an integer. If $f^{n}(f-1)^{m} f^{\prime}$ and $g^{n}(g-1)^{m} g^{\prime}$ share z CM then $f \equiv g$. 
For $m=1, n \geq 7$ we get Theorem $\mathrm{H}$.

For $m=2, n \geq 8$, we get new result.

Fang-Fang discussed Theorem $\mathrm{H}$ by replacing CM with IM and proved the following Theorem.

Theorem I ([6]). Let $f$ and $g$ be two nonconstant entire functions, $n$ be a positive integer. If $f^{n}(f-1) f^{\prime}$ and $g^{n}(g-1) g^{\prime}$ share 1 IM and $n \geq 17$, then $f \equiv g$.

We generalise the above results and prove the following Theorem.

Theorem 1.4 Let $f$ and $g$ be two transcendental entire functions, $n \geq m+15$ an integer. If $f^{n}(f-1)^{m} f^{\prime}$ and $g^{n}(g-1)^{m} g^{\prime}$ share $z$ IM then $f \equiv g$.

For $m=1, n \geq 16$ which improves Theorem I.

For $m=2, n \geq 17$, we get new result.

\section{Some Lemmas}

Lemma 2.1 ([9]) Let $f$ be a nonconstant meromorphic function, $n$ be a positive integer.

$P(f)=a_{n} f^{n}+a_{n-1} f^{n-1}+\cdots+a_{1} f$ where $a_{i}$ is a meromorphic function satisfying $T\left(r, a_{i}\right)=S(r, f)$ $(i=1,2,3, \cdots, n)$. Then

$$
T(r, P(f))=n T(r, f)+S(r, f) .
$$

Lemma 2.2 ([10]) Let $f$ be a non-constant meromorphic function $k$ be a positive integer, then

$$
N_{p}\left(r, \frac{1}{f^{(k)}}\right) \leq N_{p+k}\left(r, \frac{1}{f}\right)+k \bar{N}(r, f)+S(r, f),
$$

where $N_{p}\left(r, \frac{1}{f^{(k)}}\right)$ denotes the counting function of the zero's of $\frac{1}{f^{(k)}}$ where a zero of multiplicity $m$ is counted $m$ times if $m \leq p$ and $p$ times if $m>p$. Clearly $\bar{N}\left(r, \frac{1}{f^{(k)}}\right)=N_{1}\left(r, \frac{1}{f^{(k)}}\right)$.

Lemma 2.3 ([11] [12]) Let $F$ and $G$ be two nonconstant meromorphic functions sharing the value 1 IM. Let

$$
H=\left(\frac{F^{\prime \prime}}{F^{\prime}}-2 \frac{F^{\prime}}{F-1}\right)-\left(\frac{G^{\prime \prime}}{G^{\prime}}-2 \frac{G^{\prime}}{G-1}\right) .
$$

If $H \neq 0$, then

$$
\begin{aligned}
T(r, F)+T(r, G) \leq & 2\left[N_{2}(r, F)+N_{2}(r, G)+N_{2}\left(r, \frac{1}{F}\right)+N_{2}\left(r, \frac{1}{G}\right)\right] \\
& +3\left[\bar{N}(r, F)+\bar{N}(r, G)+\bar{N}\left(r, \frac{1}{F}\right)+\bar{N}\left(r, \frac{1}{G}\right)\right]+S(r, F)+S(r, G) .
\end{aligned}
$$

Lemma 2.4 ([5]) Let $f$ and $g$ be two nonconstant meromorphic functions, $m<7, n>7-m$ positive integers, $\alpha(z)$ denotes as in section 1 and $\alpha \neq 0, \infty$, and let

$$
F=f^{n}(f-1)^{m} f^{\prime}, \quad G=g^{n}(g-1)^{m} g^{\prime}
$$

if $F$ and $G$ share $\alpha(z) I M$, then $S(r, f)=S(r, g)$.

Lemma 2.5 ([13]) Let $H$ be defined as above. If $H \equiv 0$ and

$$
\limsup _{r \rightarrow \infty} \frac{\bar{N}\left(r, \frac{1}{F}\right)+\bar{N}(r, F)+\bar{N}\left(r, \frac{1}{G}\right)+\bar{N}(r, G)}{T(r)}<1, r \in I,
$$

where $T(r)=\max \{T(r, F), T(r, G)\}$ and $I$ is a set with infinite linear measure, then $F \equiv G$ or $F G \equiv 1$.

Lemma 2.6 ([14]) Let $Q(w)=(n-1)^{2}\left(w^{n}-1\right)\left(w^{n-2}-1\right)-n(n-2)\left(w^{n-1}-1\right)^{2}$, then

$$
Q(w)=(w-1)^{4}\left(w-\beta_{1}\right)\left(w-\beta_{2}\right) \cdots\left(w-\beta_{2 n-6}\right)
$$


where $\beta_{j} \in C \backslash\{0,1\} \quad(j=1,2,3, \cdots, 2 n-6)$, which are distinct respectively.

\section{Proofs of the Theorems}

In this section, we present the proofs of the main results.

Proof of Theorem 1.2.

Lemma 2.4 implies that $S(r, f)=S(r, g)$.

Let

$$
\begin{aligned}
& F=\frac{f^{n}(f-1)^{m} f^{\prime}}{z}, \\
& G=\frac{g^{n}(g-1)^{m} g^{\prime}}{z} .
\end{aligned}
$$

and

$$
\begin{aligned}
F^{*} & =\frac{1}{n+m+1} f^{n+m+1}-\frac{{ }^{m} C_{1}}{n+m} f^{n+m}+\frac{{ }^{m} C_{2}}{n+m-1} f^{n+m-1}+\cdots+(-1)^{p} \frac{1}{n+1} f^{n+1}, \\
G^{*} & =\frac{1}{n+m+1} g^{n+m+1}-\frac{{ }^{m} C_{1}}{n+m} g^{n+m}+\frac{{ }^{m} C_{2}}{n+m-1} g^{n+m-1}+\cdots+(-1)^{p} \frac{1}{n+1} g^{n+1},
\end{aligned}
$$

where $p=0,1,2, \cdots$

Thus we obtain that $F$ and $G$ share the value 1 IM. Moreover, by Lemma 2.1, we have

$$
\begin{gathered}
T\left(r, F^{*}\right)=(n+m+1) T(r, f)+S(r, f), \\
T\left(r, G^{*}\right)=(n+m+1) T(r, g)+S(r, g) .
\end{gathered}
$$

Noting that $\left(F^{*}\right)^{\prime}=F z$, we deduce

$$
m\left(r, \frac{1}{F^{*}}\right) \leq m\left(r, \frac{1}{z F}\right)+S(r, f) \leq m\left(r, \frac{1}{F}\right)+\log r+S(r, f),
$$

and by the First Fundamental Theorem,

$$
T\left(r, F^{*}\right) \leq T(r, F)+N\left(r, \frac{1}{F^{*}}\right)-N\left(r, \frac{1}{F}\right)+\log r+S(r, f) .
$$

Note that,

$$
N\left(r, \frac{1}{F^{*}}\right)=(n+1) N\left(r, \frac{1}{f}\right)+N\left(r, \frac{1}{f-a_{1}}\right)+N\left(r, \frac{1}{f-a_{2}}\right)+\cdots+N\left(r, \frac{1}{f-a_{m}}\right),
$$

where $a_{1}, a_{2}, \cdots, a_{m}$ are distinct roots of the algebraic equation

$$
\frac{{ }^{m} C_{0}}{n+m+1} Z^{m}-\frac{{ }^{m} C_{1}}{n+m} Z^{m-1}+\frac{{ }^{m} C_{2}}{n+m-1} Z^{m-2}+\cdots+(-1)^{p} \frac{1}{n+1}=0,
$$

and

$$
N\left(r, \frac{1}{F}\right)=n N\left(r, \frac{1}{f}\right)+N\left(r, \frac{1}{(f-1)^{m}}\right)+N\left(r, \frac{1}{f^{\prime}}\right) .
$$

Since $F$ and $G$ share 1 IM, by Lemma 2.3, we have

$$
\begin{aligned}
T(r, F)+T(r, G) \leq & 2\left[N_{2}(r, F)+N_{2}(r, G)+N_{2}\left(r, \frac{1}{F}\right)+N_{2}\left(r, \frac{1}{G}\right)\right] \\
& +3\left[\bar{N}(r, F)+\bar{N}(r, G)+\bar{N}\left(r, \frac{1}{F}\right)+\bar{N}\left(r, \frac{1}{G}\right)\right]+S(r, F)+S(r, G) .
\end{aligned}
$$


Obviously, we have

$$
\begin{aligned}
& N_{2}(r, F)+N_{2}\left(r, \frac{1}{F}\right) \leq 2 \bar{N}(r, f)+2 N\left(r, \frac{1}{f}\right)+N\left(r, \frac{1}{(f-1)^{m}}\right)+N\left(r, \frac{1}{f^{\prime}}\right)+\log r, \\
& N_{2}(r, G)+N_{2}\left(r, \frac{1}{G}\right) \leq 2 \bar{N}(r, g)+2 N\left(r, \frac{1}{g}\right)+N\left(r, \frac{1}{(g-1)^{m}}\right)+N\left(r, \frac{1}{g^{\prime}}\right)+\log r .
\end{aligned}
$$

So, we have

$$
\begin{aligned}
T\left(r, F^{*}\right)+T\left(r, G^{*}\right) \leq & T(r, F)+T(r, G)+N\left(r, \frac{1}{F^{*}}\right)+N\left(r, \frac{1}{G^{*}}\right)-N\left(r, \frac{1}{F}\right) \\
& -N\left(r, \frac{1}{G}\right)+2 \log r+S(r, f)+S(r, g) .
\end{aligned}
$$

From (5) to (14), we have

$$
(n-m-25) T(r, f)+(n-m-25) T(r, g) \leq 6 \log r+S(r, f)+S(r, g) \text {. }
$$

We obtain that $n \leq m+25$ which contradicts $n>m+26$.

Therefore $H \equiv 0$, that is

$$
\frac{F^{\prime \prime}}{F^{\prime}}-2 \frac{F^{\prime}}{F-1} \equiv \frac{G^{\prime \prime}}{G^{\prime}}-2 \frac{G^{\prime}}{G-1}
$$

By integration, we have

$$
\frac{A}{F-1}+B=\frac{1}{G-1}
$$

where $A(\neq 0)$ and $B$ are constants. Thus

$$
T(r, F)=T(r, G)+S(r, f) .
$$

Since,

$$
\bar{N}\left(r, \frac{1}{f^{\prime}}\right) \leq T\left(r, f^{\prime}\right)-m\left(r, \frac{1}{f^{\prime}}\right) \leq 2 T(r, f)-m\left(r, \frac{1}{f^{\prime}}\right)+S(r, f),
$$

we note that,

$$
\begin{aligned}
& \bar{N}\left(r, \frac{1}{F}\right)+\bar{N}\left(r, \frac{1}{G}\right)+\bar{N}(r, F)+\bar{N}(r, G) \\
& \leq \bar{N}\left(r, \frac{1}{f}\right)+\bar{N}\left(r, \frac{1}{g}\right)+\bar{N}(r, f)+\bar{N}(r, g)+\bar{N}\left(r, \frac{1}{(f-1)^{m}}\right) \\
& +\bar{N}\left(r, \frac{1}{(g-1)^{m}}\right)+\bar{N}\left(r, \frac{1}{f^{\prime}}\right)+\bar{N}\left(r, \frac{1}{g^{\prime}}\right)+2 \log r+S(r, f),
\end{aligned}
$$

and

$$
T(r, F)+m\left(r, \frac{1}{f^{\prime}}\right)=T\left(r, \frac{f^{n}(f-1)^{m} f^{\prime}}{z}\right)+m\left(r, \frac{1}{f^{\prime}}\right) \geq T\left(r, f^{n}(f-1)^{m}\right)-\log r .
$$

Similarly, we have

$$
T(r, G)+m\left(r, \frac{1}{g^{\prime}}\right) \geq T\left(r, g^{n}(g-1)^{m}\right)-\log r
$$


From (19) to (22) and applying Lemma 2.5, we get

$$
F \equiv G \quad \text { or } \quad F G \equiv 1 .
$$

We discuss the following cases.

Case (i) Suppose that $F G \equiv 1$.

As in the proof of Theorem 1, in [5] we arrive at a contradiction.

Case (ii) $F \equiv G$, thus $F^{*} \equiv G^{*}$, that is,

$$
\begin{aligned}
& \frac{1}{n+m+1} f^{n+m+1}-\frac{{ }^{m} C_{1}}{n+m} f^{n+m}+\frac{{ }^{m} C_{2}}{n+m-1} f^{n+m-1}+\cdots+(-1)^{p} \frac{1}{n+1} f^{n+1} \\
& =\frac{1}{n+m+1} g^{n+m+1}-\frac{{ }^{m} C_{1}}{n+m} g^{n+m}+\frac{{ }^{m} C_{2}}{n+m-1} g^{n+m-1}+\cdots+(-1)^{p} \frac{1}{n+1} g^{n+1} .
\end{aligned}
$$

Set $h=\frac{f}{g}$, we substitute $f=h g$ in the above, it follows that

$$
\begin{aligned}
& (n+m)(n+m-1) \cdots(n+1) g^{m}\left(h^{n+m+1}-1\right)-{ }^{m} C_{1}(n+m+1)(n+m-1) \cdots \\
& (n+1) g^{m-1}\left(h^{n+m}-1\right)+\cdots+(-1)^{p}(n+m+1)(n+m) \cdots(n)\left(h^{n+1}-1\right)=0 .
\end{aligned}
$$

If $h$ is not a constant, using Lemma 2.6 and (23), we conclude that

$$
\begin{aligned}
& \left.(n+m)(n+m-1) \cdots(n+1) g\left(h^{n+m+1}-1\right)-(n+m+1)(n+m-1) \cdots(n+1)\left(h^{n+m}-1\right)\right]^{m} \\
= & (n+m)^{m}(n+m-1)^{m} \cdots(n+1)^{m}\left(h^{n+m+1}-1\right)^{m} g^{m}-{ }^{m} C_{1}(n+m)^{m-1}(n+m-1)^{m-1} \cdots \\
& (n+1)^{m-1}(n+m+1)(n+m-1) \cdots(n+1)\left(h^{n+m+1}-1\right)^{m-1}\left(h^{n+m}-1\right) g^{m-1} \\
& +\cdots+(-1)^{p}(n+m+1)^{m}(n+m-1)^{m} \cdots(n+1)^{m}\left(h^{n+m}-1\right)^{m} \\
= & (n+m)^{m-1}(n+m-1)^{m-1} \cdots(n+1)^{m-1}\left(h^{n+m+1}-1\right)^{m-1}\{(n+m)(n+m-1) \cdots \\
& \left.(n+1) g^{m}\left(h^{n+m+1}-1\right)-{ }^{m} C_{1}(n+m+1)(n+m-1) \cdots(n+1) g^{m-1}\left(h^{n+m}-1\right)+\cdots\right\} \\
& +(-1)^{p}(n+m+1)^{m}(n+m-1)^{m} \cdots(n+1)^{m}\left(h^{n+m}-1\right)^{m}
\end{aligned}
$$

By (23), we get

$$
\begin{aligned}
= & (n+m)^{m-1}(n+m-1)^{m-1} \cdots(n+1)^{m-1}\left(h^{n+m+1}-1\right)^{m-1}\{-(n+m+1)(n+m) \cdots \\
& \left.\left(h^{n+1}-1\right)\right\}+(n+m+1)^{m}(n+m-1)^{m} \cdots(n+1)^{m}\left(h^{n+m}-1\right)^{m} \\
= & (n+m+1)(n+m-1)^{m-1} \cdots(n+1)^{m-1}\left\{(n+m)^{m}\left(h^{n+m+1}-1\right)^{m-1}\left(h^{n+1}-1\right)\right. \\
& \left.-(n+m+1)^{m-1}(n+m-1) \cdots(n+1)^{m}\left(h^{n+m}-1\right)^{m}\right\} \\
= & (n+m+1)(n+m-1)^{m-1} \cdots(n+1)^{m-1} Q(h),
\end{aligned}
$$

where

$$
Q(h)=(n+m)^{m}\left(h^{n+m+1}-1\right)^{m-1}\left(h^{n+1}-1\right)-(n+m+1)^{m-1}(n+m-1) \cdots(n+1)^{m}\left(h^{n+m}-1\right)^{m} .
$$

Using Lemma 2.6, we get

$$
Q h=(h-1)^{4}\left(h-\beta_{1}\right)\left(h-\beta_{2}\right) \cdots\left(h-\beta_{2 n+2 m-4}\right) .
$$

where $\beta_{j} \in C \backslash\{0,1\},(j=1,2, \cdots, 2 n+2 m-4)$ which are pairwise distinct.

This implies that every zero of $h-\beta_{j} \quad(j=1,2, \cdots, 2 n)$ has a multiplicity of at least $n$. By the Second Fun- 
damental Theorem, we obtain that $n \leq m$, which is again a contradiction.

Therefore $h$ is a constant. We have from (23) that $h^{n+1}-1=0, h^{n+m}-1=0$, which imply $h=1$, and hence $f \equiv g$.

Proof of Theorem 1.1. Let $F$ and $G$ be given by (1) and (2). Suppose $H$ is given as in Lemma 2.3, and $H \neq 0$. Proceeding as in the proof of Theorem 1.2 we can obtain (3) to (10). Since $F$ and $G$ share $1 C M$, by Lemma 2.3, we have

$$
T(r, f) \leq N_{2}(r, F)+N_{2}(r, G)+N_{2}\left(r, \frac{1}{F}\right)+N_{2}\left(r, \frac{1}{G}\right) .
$$

Hence from (3) to (10) and (12) to (14), we get

$$
(n-m-10) T(r, f)+(n-m-10) T(r, g) \leq S(r, f)+S(r, g) .
$$

Hence $n \leq m+10$, which contradicts that $n \geq m+11$.

Proof of Theorem 1.4. Let $F$ and $G$ be given by (1) and (2). Suppose $H$ is given as in Lemma 2.3, and $H \neq 0$. Proceeding as in the proof of Theorem 1.2 we can obtain (3) to (10) and (11). Since $F$ and $G$ share 1 IM, by Lemma 2.3, obviously we have,

$$
\begin{aligned}
& N_{2}(r, F)+N_{2}\left(r, \frac{1}{F}\right) \leq 2 N\left(r, \frac{1}{f}\right)+N\left(r, \frac{1}{(f-1)^{m}}\right)+N\left(r, \frac{1}{f^{\prime}}\right), \\
& N_{2}(r, G)+N_{2}\left(r, \frac{1}{G}\right) \leq 2 N\left(r, \frac{1}{g}\right)+N\left(r, \frac{1}{(g-1)^{m}}\right)+N\left(r, \frac{1}{g^{\prime}}\right) .
\end{aligned}
$$

therefore (14) reduces to

$$
(n-m-14) T(r, f)+(n-m-14) T(r, g) \leq S(r, f)+S(r, g) .
$$

Hence $n \leq m+14$, which contradicts that $n \geq m+15$. Proceeding in the same way as in Theorem 1.2 we get $f \equiv g$.

Proof of Theorem 1.3. Let $F$ and $G$ be given by (1) and (2). Suppose $H$ is given as in Lemma 2.3, and $H \neq 0$. Proceeding as in the proof of Theorem 1.2 we can obtain (3) to (10). Since $F$ and $G$ share $1 C M$, by Lemma 2.3, we have

$$
T(r, F) \leq N_{2}(r, F)+N_{2}\left(r, \frac{1}{F}\right)+N_{2}(r, G)+N_{2}\left(r, \frac{1}{G}\right) .
$$

Hence from (3) to (10) and (12) to (14), we get

$$
(n-m-5) T(r, f)+(n-m-5) T(r, g) \leq S(r, f)+S(r, g) .
$$

Hence $n \leq m+5$, which contradicts that $n \geq m+6$. Proceeding in the same way as in Theorem 1.2, we get $f \equiv g$.

\section{Acknowledgements}

The author would like thank Prof. S. S. Bhoosnurmath for his valuable suggestions for the improvement of the paper. This research was supported by the UGC under MRP(S).

\section{References}

[1] Hayman, W.K. (1964) Meromorphic Functions. Clarendon Press, Oxford.

[2] Yang, C.C. and Hua, X.H. (1997) Uniqueness and Value-Sharing of Meromorphic Functions. Annales Academice Scientiarum Fennicce Mathematica, 22, 395-406.

[3] Fang, M.L. and Hong, W. (2001) A Unicity Theorem for Entire Functions Concerning Differential Polynomials, Indian. Journal of Pure and Applied Mathematics, 32, 1343-1348.

[4] Lin, W.C. and Yi, H.X. (2004) Uniqueness Theorem for Meromorphic Functions Concerning Fixed Points. Complex Variables, Theory and Application, 49, 793-806. 
[5] Chao, M. (2008) Uniqueness Theorems for Differential Polynomials Concerning Fixed Points. Kyungpook Mathematical Journal, 48, 25-35. http://dx.doi.org/10.5666/KMJ.2008.48.1.025

[6] Fang, C.Y. and Fang, M.L. (2002) Uniqueness of Meromorphic Functions and Differential Polynomials. Computers \& Mathematics with Applications, 44, 607-617. http://dx.doi.org/10.1016/S0898-1221(02)00175-X

[7] Lin, W.C. and Yi, H.X. (2004) Uniqueness Theorems for Meromorphic Functions. Indian Journal of Pure and Applied Mathematics, 35, 121-132.

[8] Qiu, H.L. and Fang, M.L. (2004) On the Uniqueness of Entire Functions. Bulletin of the Korean Mathematical Society, 41, 109-116. http://dx.doi.org/10.4134/BKMS.2004.41.1.109

[9] Yang, C.C. and Yi, H.X. (2003) Uniqueness Theory of Meromorphic Functions. Kluwer Academic Publishers, London.

[10] Zhang, Q.C. (2005) Meromorphic Functions That Share One Small Functions with Its Derivatives. Journal of Inequalities in Pure and Applied Mathematics, 6, Article ID: 116.

[11] Xu, J.F. and Yi, H.X. (2007) Uniqueness of Entire Functions and Differential Polynomials. Bulletin of the Korean Mathematical Society, 44, 623-629. http://dx.doi.org/10.4134/BKMS.2007.44.4.623

[12] Yi, H.X. (1999) Meromorphic Functions That Share One or Two Values II. Kodai Mathematical Journal, 22, $264-272$. http://dx.doi.org/10.2996/kmj/1138044046

[13] Yi, H.X. (1995) Meromorphic functions That Share One or Two Values. Complex Variables, Theory and Application, 28, 1-11.

[14] Frank, G. and Reiders, M. (1998) A Unique Range Set for Meromorphic Functions with 11 Elements. Complex Variables, Theory and Application, 37, 185-193. 